\title{
Multiband photometric decomposition of nuclear stellar disks
}

\author{
L. Morelli ${ }^{1}$, M. Cesetti ${ }^{2}$, E. M. Corsini ${ }^{1}$, A. Pizzella ${ }^{1}$, E. Dalla Bontà ${ }^{1}$, M. Sarzi $^{3}$, and F. Bertola ${ }^{1}$ \\ 1 Dipartimento di Astronomia, Università di Padova, vicolo dell'Osservatorio 3, 35122 Padova, Italy \\ e-mail: lorenzo.morelli@unipd.it \\ 2 INAF-Osservatorio Astronomico di Padova, vicolo dell'Osservatorio 2, 35122 Padova, Italy \\ 3 Centre for Astrophysics Research, University of Hertfordshire, College Lane, Hatfield, Herts AL10 9AB, UK
}

Received 18 February 2010 / Accepted 6 April 2010

\section{ABSTRACT}

\begin{abstract}
Context. Small, bright stellar disks with scale lengths of a few tens of parsec are known to reside in the center of galaxies. They are believed to have formed in a dissipational process as the end result of star formation in gas either accreted during a merging (or acquisition) event or piled up by the secular evolution of a nuclear bar. Only a few of them have been studied in detail to date.

Aims. Using archival Hubble Space Telescope (HST) imaging, we investigate the photometric parameters of the nuclear stellar disks hosted by three early-type galaxies in the Virgo cluster, NGC 4458, NGC 4478, and NGC 4570, to constrain the process that forms their stars.

Methods. The central surface brightness, scale length, inclination, and position angle of the nuclear disks were derived by adopting the photometric decomposition method introduced by Scorza \& Bender and assuming the disks to be infinitesimally thin and exponential.

Results. The location, orientation, and size of the nuclear disks is the same in all the images obtained with the Wide Field Planetary Camera 2 and Advanced Camera for Surveys and available in the HST Science Archive. The scale length, inclination, and position angle of each disk are constant within the errors in the observed $U, B, V$, and $I$ passbands, independently of their values and the properties of the host spheroid.

Conclusions. We interpret the absence of color gradients in the stellar population of the nuclear disks as the signature that star formation homogeneously occurred along their length. An inside-out formation scenario is, instead, expected to produce color gradients and is therefore ruled out.
\end{abstract}

Key words. Galaxy: bulge - galaxies: elliptical and lenticular, cD - galaxies: photometry - galaxies: structure

\section{Introduction}

The presence of small and bright stellar disks with scale lengths of a few tens of parsec was first discovered in the nuclear regions of nearby galaxies about fifteen years ago thanks to the subarcsec-resolution capabilities of the Hubble Space Telescope (HST, van den Bosch et al. 1994; Kormendy et al. 1994; Emsellem et al. 1994; Lauer et al. 1995). Afterward, nuclear stellar disks (NSDs) and larger scale embedded disks were reported in a large number of early-type galaxies as a results of photometric (Lauer et al. 1995; Carollo et al. 1997; Ravindranath et al. 2001; Rest et al. 2001; Tran et al. 2001; Ferrarese et al. 2006; Seth et al. 2006; Simões Lopes et al. 2007; Kormendy et al. 2009) and spectroscopic surveys (Halliday et al. 2001; Kuntschner et al. 2006; McDermid et al. 2006) respectively. Given that nuclear disks are easiest to detect when nearly edgeon (Rix \& White 1990), the observed fraction is consistent with NSDs being a common structure in early-type galaxies (Ledo et al. 2010). At the center of spiral galaxies NSDs are also present but these are relatively rare in late types (Pizzella et al. 2002; Falcón-Barroso et al. 2006; Peletier et al. 2007; Balcells et al. 2007; Morelli et al. 2008).

Photometric parameters of NSDs (i.e., central surface brightness, scale-length, axial ratio, and position angle) have been derived for nine galaxies spanning a wide range of Hubble types, from ellipticals (Morelli et al. 2004), to lenticulars (van den Bosch et al. 1994; Lauer et al. 1995; Kormendy et al. 1996; van den Bosch et al. 1998; Scorza \& van den Bosch 1998), and to early-type spirals (Pizzella et al. 2002). They are characterized by a smaller scale length $(10-30 \mathrm{pc})$ and higher central face-on surface brightness (15-19 mag $\operatorname{arcsec}^{-2}$ in the $V$ band) than the embedded stellar disks observed in ellipticals and lenticulars as well as to the large kpc-scale disks of lenticulars and spirals (van den Bosch 1998; Pizzella et al. 2002; Morelli et al. 2004). In particular, the embedded stellar disks represent the intermediary between the sequence of elliptical galaxies and that of disk galaxies. They have been detected in both disky elliptical and lenticular galaxies, have scale lengths of $0.1-1 \mathrm{kpc}$, and central face-on surface brightnesses of 18-21 mag $\operatorname{arcsec}^{-2}$ in the $V$ band. Their angular momentum is parallel to that of their host spheroid. This demonstrates that the embedded disks are not the result of accretion or merger events but are likely to be primordial, in the same sense as the main disks of lenticular and spiral galaxies (Scorza \& Bender 1995). The smooth variation in the scale parameters from the nuclear to the embedded and main stellar galactic disks provides observational support that galaxy properties vary with continuity along a sequence of increasing disk-to-bulge ratio (see Kormendy \& Bender 1996, and references therein). Therefore, unveiling the formation scenario of NSDs may also improve our understanding of galaxy formation and evolution.

In the current picture, NSDs are believed to have formed in a dissipational process as the end result of star formation in gas either accreted in a merging (or acquisition) event (the most clearcut case is NGC 4698, Bertola et al. 1999; Pizzella et al. 2002) or piled up by the secular evolution of a nuclear bar (e.g., 
NGC 4570, van den Bosch et al. 1998; Scorza \& van den Bosch 1998; van den Bosch \& Emsellem 1998). Each of these scenarios is likely to be correct for some, but not all, objects. In both of them, the gas is efficiently directed toward the galaxy center (e.g., Barnes \& Hernquist 1996; Athanassoula et al. 2005; Dotti et al. 2007; Eliche-Moral et al. 2009; Hopkins \& Quataert 2010), where it first dissipates and settles onto an equilibrium plane and then forms into stars. A striking example of an on going process of dissipational formation is provided by NGC 4486A (Kormendy et al. 2005). The NSD of this low-luminosity elliptical coexists with its progenitor disk of dust and gas. The question about the origin of these dynamically-cold components is also related to their formation epoch, i.e., whether they built up in the early stages of the galaxy assembly or whether they formed later.

To address this issue, van den Bosch et al. (1998), van den Bosch \& Emsellem (1998), Morelli et al. (2004), and Krajnović \& Jaffe (2004) studied the age and metallicity content of some NSDs. In some cases, the nuclear disk was found to be younger than the stellar population of their surrounding spheroidal component (e.g., NGC 4486A, Kormendy et al. 2005), in other cases the formation of the disks occurred at the same time as the main body of the host galaxy (e.g., NGC 4342, van den Bosch et al. 1998).

Characterizing how the star formation proceeded in nuclear disks can lead to understanding what triggered their formation. For instance, if stars formed more or less at the same rate throughout the disk from disk material that accumulated very rapidly at the center, as one expects to happen during a merging event, we should not find strong radial gradients in the properties of the stellar populations. In contrast, color gradients are expected if the disk experiences an inside-out star formation, like the disks of spiral galaxies (e.g., Muñoz-Mateos et al. 2007). In this paper, we use HST archival images of the nuclei of three early-type galaxies in the Virgo cluster, NGC 4458, NGC 4478, and NGC 4570 to perform a multiband analysis of the photometric parameters of their NSDs. Our aim is to understand whether the star formation of the nuclear disk was homogeneous and occurred simultaneously everywhere along its length.

\section{Sample galaxies}

We searched the HST Science Archive for all NSDs known in literature that had been imaged in at least three filters with the Wide Field Planetary Camera 2 (WFPC2) and Advanced Camera for Surveys (ACS) and had known properties of their stellar population. We found multiband images of the nuclear disks hosted in three early-type galaxies of the Virgo cluster, NGC 4458, NGC 4478, and NGC 4570 (Table 1).

NGC 4458 and NGC 4478 are classified as E0-1 (de Vaucouleurs et al. 1991, hereafter RC3) and E2 (Sandage \& Tammann 1981, hereafter RSA; RC3), respectively. They are low-luminosity ellipticals with a $M_{B}^{0} \sim-18$ (RC3) at a distance of $12.6 \mathrm{Mpc}$ (Tully 1988, $H_{0}=100 \mathrm{~km} \mathrm{~s}^{-1} \mathrm{Mpc}^{-1}$ ). Both galaxies host a kinematically-decoupled core (Halliday et al. 2001; Emsellem et al. 2007). Morelli et al. (2004) detected NSDs inside both on the same radial scale as the core and analyzed their structure and stellar content. Their scale length and face-on central surface brightness (Table 2) were derived from WFPC2/F814W images and they fit in the relation for NSDs. For NGC 4458, these parameters are typical of NSDs, while for NGC 4478 they are in-between those of NSDs and the disks of disky ellipticals. The mass of the two disks is of few $10^{7} M_{\odot}$. The central disk of NGC 4458 is counter-rotating. It has similar properties to the decoupled cores of bright ellipticals (see Bertola \& Corsini 1999, for a review). According to Morelli et al. (2004), it is as old as $(t \simeq 15 \mathrm{Gyr})$ and richer in metals $([Z / H] \simeq 0.2)$ than the rest of the galaxy, with a high and approximately constant overabundance $([\alpha / \mathrm{Fe}] \simeq 0.3)$. The NSD of NGC 4478 is co-rotating with respect to the main body of its host galaxy, but it is younger $(t \simeq 6 \mathrm{Gyr})$, richer in metals $([Z / \mathrm{H}] \simeq 0.4)$ and less overabundant $([\alpha / \mathrm{Fe}] \simeq 0.2)$ than the outer regions. The nearly solar $\alpha$-element abundance indicates a prolonged star formation history, typical of an undisturbed disklike, gas-rich, and possibly pre-enriched structure. Morelli et al. (2004) used the models of single stellar populations with $\alpha / \mathrm{Fe}$ overabundances developed by Thomas et al. (2003) to derive the properties of the nuclear stellar populations. They analyzed the absorption line-strength indices measured along the major and minor axes of both galaxies. The masses of the two NSDs were estimated by adopting the mass-to-light ratios calculated with the models of Maraston (1998) from the available ages and metallicities. The presence of cold disks at the centers of NGC 4458 and NGC 4478 argues for a formation scenario based on the dissipational collapse of a gas-rich object. An external origin of the gas is claimed to account for the counter-rotation of NGC 4478 (Morelli et al. 2004).

NGC 4570 is a edge-on disk galaxy classified as S0 by RSA and RC3. It has $M_{B}^{0} \sim-19(\mathrm{RC} 3)$ at a distance of $12.6 \mathrm{Mpc}$ (Tully 1988). Its nuclear disk was first studied in detail by van den Bosch et al. (1998). It resides within the inner cutoff of the main stellar disk, whose light contribution to the galaxy surface brightness is negligible within the inner $2^{\prime \prime}$. The inner truncation of the main disk is a general feature in multi-disk systems hosting a nuclear (van den Bosch et al. 1998; Pizzella et al. 2002) and/or an embedded disk (e.g., Scorza \& Bender 1995; Seifert \& Scorza 1996; Emsellem et al. 1996). The photometric parameters (Table 2) were derived by analyzing a WFPC2/F555W image and are typical of NSDs (Scorza \& van den Bosch 1998). The NSD of NGC 4570 has a mass of $8 \times 10^{7} M_{\odot}$ (at the adopted distance) as derived from dynamical modelling by van den Bosch \& Emsellem (1998). It has a different stellar population than the rest of the bulge, consisting of relatively old $(t \simeq 8 \mathrm{Gyr})$ and metal-rich $([\mathrm{Fe} / \mathrm{H}] \simeq 0.35)$ stars (van den Bosch et al. 1998). These results were derived by comparing the colors measured in WFPC2 images and the line-strength indices measured in Faint Object Spectrograph spectra with the models of single stellar populations by Worthey (1994).

In addiction, NGC 4570 exhibits two nuclear edge-on rings. According to dynamical modelling by van den Bosch \& Emsellem (1998) based on the observed surface brightness and stellar kinematics, the position of the rings (at about $2^{\prime \prime}$ and 4 " from the center, respectively) is consistent with the location of the inner Lindblad and ultra-harmonic resonances of a rapidlyrotating nuclear bar of about $500 \mathrm{pc}$. The corotation radius is close to the inner cut-off of the main disk at about 7 " from the center. The morphology, dynamics, star formation, and chemical enrichment of the nuclear region strongly favor a formation scenario of the NSD driven by the secular evolution. The nuclear disk appears to be the end result of the star formation that occurred in the gas that was funneled to the center by the nuclear bar (van den Bosch \& Emsellem 1998; Krajnović \& Jaffe 2004). The growth of a central mass concentration produced by the gas inflow altered the shape of the orbits sustaining the bar. As a consequence, the nuclear bar weakened and dissolved (see Athanassoula et al. 2005, and references therein). 
Table 1. Details about the HST archival images of the sample galaxies.

\begin{tabular}{ccccrrrl}
\hline \hline Galaxy & Camera & Filter & Obs. Date & No. Exp. & $\begin{array}{c}\text { Exp. Time } \\
{[\mathrm{s}]}\end{array}$ & Prog. Id. & P.I. \\
\cline { 3 - 7 }$(1)$ & $(2)$ & $(3)$ & $(4)$ & $(5)$ & $(6)$ & $(7)$ & $(8)$ \\
\hline \multirow{2}{*}{ NGC 4458 } & ACS/WFC & $F 475 W$ & 28 Dec. 2003 & 2 & 750 & 9401 & P. Côté \\
& WFPC2/PC & $F 555 W$ & 18 Dec. 1998 & 3 & 1340 & 6587 & D. O. Richstone \\
& WFPC2/PC & $F 814 W$ & 12 Feb. 1995 & 6 & 1120 & 5512 & S. M. Faber \\
NGC 4478 & ACS/HRC & $F 330 W$ & 28 Jul. 2005 & 4 & 1912 & 10435 & R. W. O'connell \\
& ACS/WFC & $F 475 W$ & 09 Jul. 2003 & 2 & 750 & 9401 & P. Côté \\
& WFPC2/PC & $F 555 W$ & 07 Jul. 1997 & 4 & 1600 & 6587 & D. O. Richstone \\
& WFPC2/PC & $F 814 W$ & 07 Jul. 1997 & 3 & 1600 & 6587 & D. O. Richstone \\
NGC 4570 & WFPC2/PC & $F 336 W$ & 19 Apr. 1996 & 5 & 3100 & 6107 & W. Jaffe \\
& ACS/WFC & $F 475 W$ & 13 Jul. 2003 & 2 & 750 & 9401 & P. Côté \\
& WFPC2/PC & $F 555 W$ & 19 Apr. 1996 & 2 & 400 & 6107 & W. Jaffe \\
& WFPC2/PC & $F 814 W$ & 19 Apr. 1996 & 2 & 460 & 6107 & W. Jaffe \\
\hline
\end{tabular}

Notes. Column (1): Galaxy name. Column (2): camera name. Column (3): filter name. Column (4): observation date. Column (5): number of exposures. Column (6): total exposure time. Column (7): HST proposal number. Column (8): name of the principal investigator.

\section{Data reduction}

The WFPC2 and ACS multiband images of NGC 4458, NGC 4478, and NGC 4570 were retrieved from the HST Science Data Archive. Their list and observing log are given in Table 1.

The WFPC2 images were obtained by centering the galaxy nucleus on the higher resolution Planetary Camera (PC). The PC detector is a Loral CCD with $800 \times 800$ pixels. The pixel size is $15 \times 15 \mu \mathrm{m}^{2}$. The plate scale of 0 ' $^{\prime} 046$ pixel $^{-1}$ yields a field of view of about $36^{\prime \prime} \times 36^{\prime \prime}$. To help in identifying and correcting cosmic-ray events, different exposures were taken with each filter. The ACS images were taken with both the Wide Field Channel (WFC) and the High Resolution Channel (HRC). WFC consists of two SITe CCDs with $2048 \times 4096$ pixels each of size $15 \times 15 \mu \mathrm{m}^{2}$. The plate scale is 0 . $^{\prime} 049$ pixel $^{-1}$ and the field of view of the combined detectors covers an approximately square area of about $202^{\prime \prime} \times 202^{\prime \prime}$. HRC detector is a SITe CCDs with $1024 \times 1024$ pixels each of size $21 \times 21 \mu \mathrm{m}^{2}$ with a plate scale of 0 . $^{\prime} 028 \times 0.0^{\prime} 025$ pixel $^{-1}$. Because of the large, but well characterized, geometric distortion affecting the ACS, the $29^{\prime \prime} \times 26^{\prime \prime}$ HRC field of view projects onto the plane of the sky as a rhomboid with $x$ and $y$ axes forming a $84^{\circ} .2$ angle. For both WFPC2 and ACS images, the telescope was guiding in fine lock, giving a typical rms tracking error per exposure of 0.005 .

The WFPC2 images were calibrated using the CalWFPC reduction pipeline in IRAF $^{1}$ maintained by the Space Telescope Science Institute. Reduction steps include bias subtraction, dark current subtraction, and flat-fielding, as described in detail in the WFPC2 instrument and data handbooks (Baggett et al. 2002; McMaster et al. 2008). Subsequent analysis was performed using IRAF standard tasks. The bad pixels were corrected by means of a linear one-dimensional interpolation using the data quality files and the WFIXUP task. For each galaxy, the alignment of the images obtained with the same filter was checked by comparing the centroids of stars in the field of view. The images were aligned to an accuracy of a few hundredths of a pixel using IMSHIFT and knowledge of the offsets. They were then combined with IMCOMBINE. We verified that the alignment and combination did not introduce a significant blurring of the

\footnotetext{
1 Imaging reduction and analysis facilities (IRAF) is distributed by the National Optical Astronomy Observatories which are operated by the Association of Universities for Research in Astronomy (AURA) under cooperative agreement with the National Science Foundation.
}

data. To this aim, we measured the FWHM of the Gaussian fitting to the field stars in the original and combined frames. We found that they did not change to within a few percent. In combining the images, pixels deviating by more than three times the local standard deviation - calculated from the combined effect of Poisson and read-out noise - were flagged as cosmic rays and rejected. The residual cosmic rays and bad pixels were corrected by manually editing the resulting image with IMEDIT.

The ACS images were calibrated using the CalACS reduction pipeline. Reduction steps include bias subtraction, dark current subtraction, flat-fielding correction, and correction for geometric distortion with MULTIDRIZZLE task of IRAF as described in detail in ACS instrument and data handbooks (Pavlovsky et al. 2004, 2006). The images obtained in the same filter were aligned by comparing the centroids of stars in the field of view and then combined, rejecting cosmic rays in the process. Residual cosmic ray events and hot pixels were removed using the LACOS_IMA procedure (van Dokkum 2001).

The sky level in WFPC2 images was determined from apparently empty regions in the Wide Field chips and subtracted from the PC frame after appropriate scaling. In ACS images, it was determined from regions free of sources at the edge of the field of view and then subtracted. The ACS/F330W and WFPC2/F336W passbands approximate Johnson-Cousins $U$ band. The ACS/F475W, WFPC2/F555W and WFPC2/F814W passbands are similar to Johnson-Cousins $B, V$, and $I$ bands, respectively. The flux calibration to the Vega magnitude system was performed following Holtzman et al. (1995) for the WFPC2 images, and Sirianni et al. (2005) for the ACS images.

\section{Unsharp images of nuclear disks}

To first gauge the structure and extent of the NSDs in all the available images of the sample galaxies, we constructed the unsharp-masked image of each frame using an identical procedure described by Pizzella et al. (2002). The ACS/HRC image was resampled to match the same spatial scale as the WFPC2/PC images. Each image was divided by itself after convolution by a circular Gaussian of width $\sigma=6$ pixels, corresponding to 0.28 and 0.'29 in the WFPC2/PC and ACS/WFC images, respectively (Fig. 1). This procedure enhanced any surface-brightness fluctuation and non-circular structure extending over a spatial region comparable to the $\sigma$ of the smoothing Gaussian. Each galaxy 


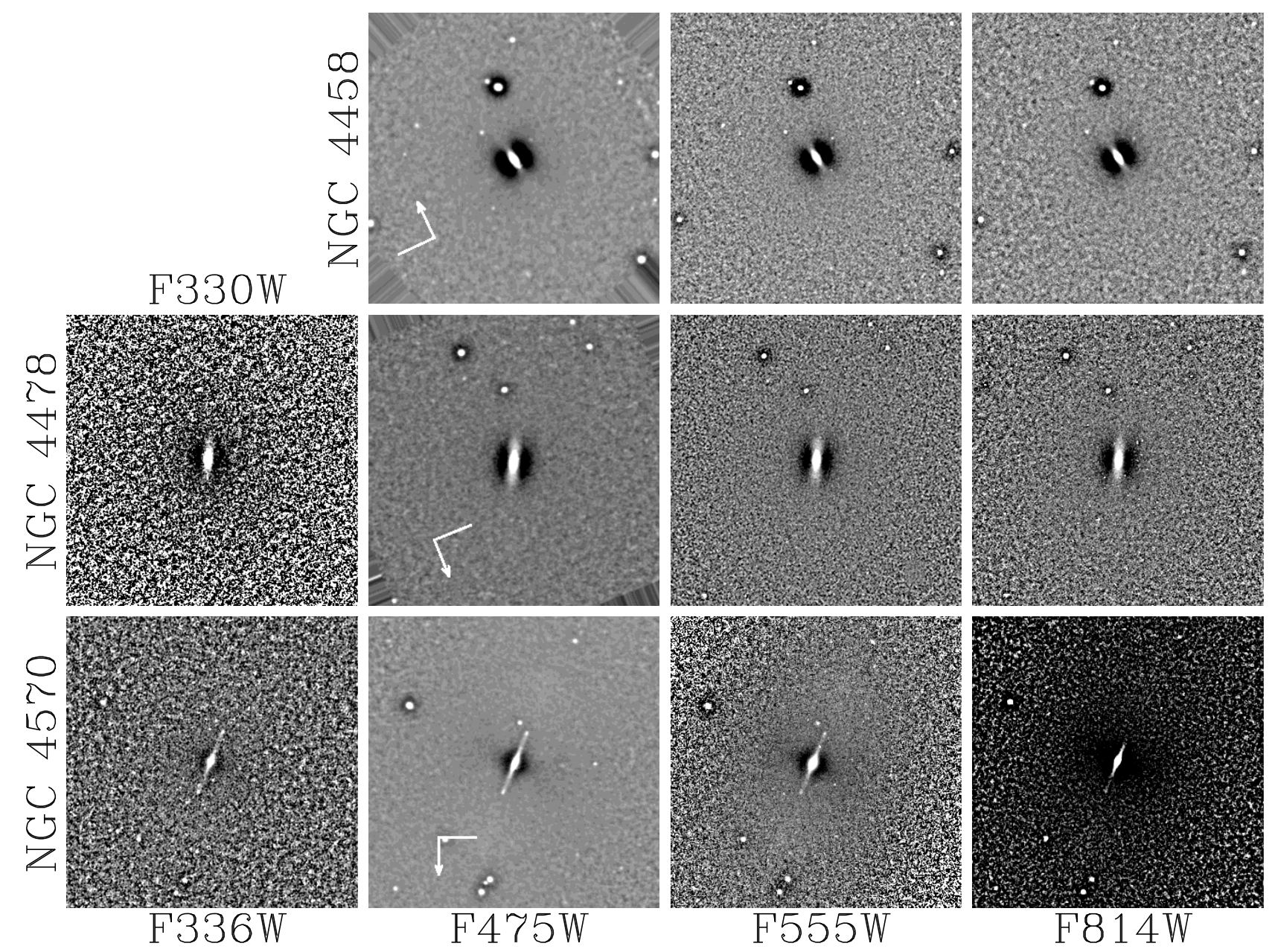

Fig. 1. Unsharp masking of the HST archival images of NGC 4458 (ACS/F475W, WFPC2/F555W, and WFPC2/F814W; top panels), NGC 4478 (ACS/F330W, ACS/F475W, WFPC2/F555W, and WFPC2/F814W; middle panels), and NGC 4570 (WFPC2/F336W, ACS/F475W, WFPC2/F555W, and WFPC2/F814W; bottom panels). The orientation is specified by the arrow indicating north and the segment indicating east in the lower-left corner of the ACS/F475W image. The size of the plotted region is about $15^{\prime \prime} \times 15^{\prime \prime}$.

nucleus clearly contains a highly elongated structure in all the images. The location, orientation, and size of each structure is similar in all the observed passbands. These nuclear structures are associated with a central increase in ellipticity measured by performing an isophotal analysis using the IRAF task ELLIPSE (Figs. 2-4; see also Scorza \& van den Bosch 1998; Morelli et al. 2004). They are not artifacts of the unsharp-masking procedure as discussed by Morelli et al. (2004) for NGC 4458 and NGC 4478 and by Scorza \& van den Bosch (1998) for NGC 4570.

The ACS/F475W unsharped-masked image of NGC 4570 exhibits two symmetric bright spots located along the major axis of the NSD at about $2^{\prime \prime}$ from the center. They were identified by van den Bosch \& Emsellem (1998) with the signature of the nuclear ring located at the inner Lindblad resonance in the equatorial plane of the galaxy. This ring is seen edge-on. The two spots are barely visible in the WFPC2/F336W and WFPC2/F555W images and almost missing in the WFPC2/F814W image. According to van den Bosch \& Emsellem (1998), the blue colors of these features correspond to a rather young stellar population with an age of less than 2 Gyr and a metallicity close to solar. The width of the filtering Gaussian we adopted in the unsharpmasking process is effective in detecting the NSD, but does not enhance the structure of the ring located at the ultra-harmonic resonance nor the inner cutoff to the main disk (for this purpose, see the unsharp-masked images obtained with different filtering Gaussians that van den Bosch \& Emsellem 1998, displayed in their Fig. 1).

In Morelli et al. (2004), we derived isophotal profiles from the analysis of the WFPC2/F814W images of NGC 4458 and NGC 4478. Here, we present the isophotal profiles derived from the remaining images of the sample galaxies adopting the same method. We first masked foreground stars and then fitted ellipses to the isophotes. We allowed the centers of the ellipses to vary, to test whether the galaxies were disturbed. Within the errors of the fits, we found no evidence of variations in the fitted center. The ellipse fitting was repeated with the ellipse centers fixed. The signal-to-noise ratio of the ACS/F330W image of NGC 4478 did not allow us to measure a reliable radial profile of the $a_{4}$ coefficient. This is necessary to derive the NSD parameters, therefore we did not consider this image in the subsequent analysis. The resulting azimuthally averaged surface brightness, ellipticity, position angle, and the fourth cosine Fourier coefficient $\left(a_{4}\right)$ profiles of NGC 4458, NGC 4478, and NGC 4570 are presented in Figs. 2, 3, and 4, respectively. In the innermost about $1^{\prime \prime}$ of NGC 4458 and NGC 4570 and about $2^{\prime \prime}$ of NGC 4478, we measured positive values of the $a_{4}$ Fourier coefficients, which describe the disky deviation of the isophotes 
L. Morelli et al.: Multiband photometric decomposition of nuclear stellar disks

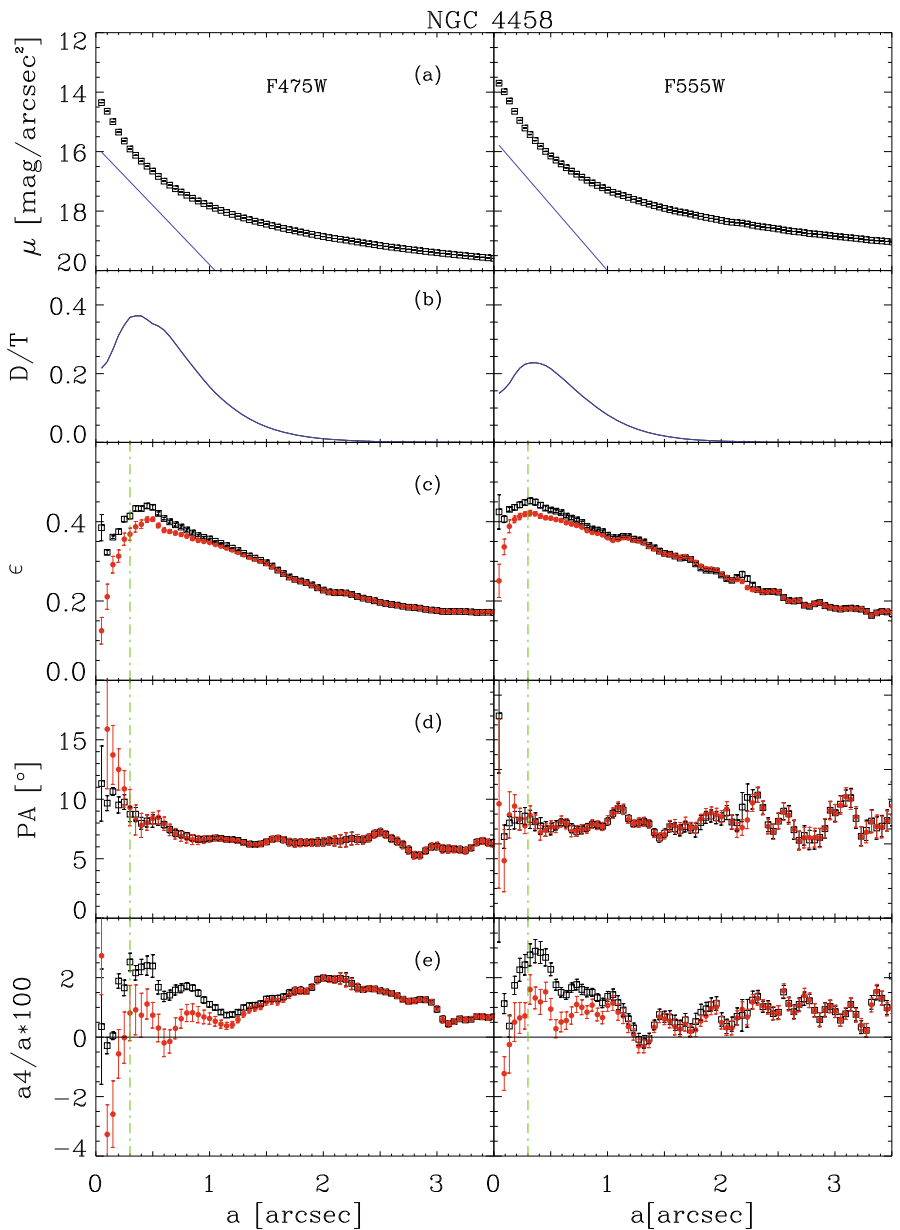

Fig. 2. Isophotal parameters of the nuclear region of NGC 4458 as a function of the isophotal semi-major axis. We show the results based on the analysis of the surface-brightness distribution measured in the $\mathrm{ACS} / \mathrm{F} 475 \mathrm{~W}$ (left panels) and WFPC2/F555W (right panels) images, respectively. a) Surface-brightness radial profiles of the galaxy after deconvolution (open black squares) and of the nuclear disk (solid blue line). b) Radial profile of the fraction of total luminosity contributed by the nuclear disk. Radial profiles of the galaxy ellipticity c), position angle d), and fourth cosine Fourier coefficient e) before (open black squares) and after (filled red circles) the subtraction of the best-fitting model for the nuclear disk. The results of the photometric decomposition of the WFPC2/F814W image are in Morelli et al. (2004). The vertical dash-dotted line indicates the radius inside which the deconvolution algorithm is not able to recover the geometric parameters of the isophotes.

from pure ellipses (Jedrzejewski 1987). These photometric features confirm the presence of a NSD (see also Kormendy et al. 2009).

\section{Structural parameters of nuclear disks}

After establishing the existence of NSDs in all passbands, we derived their photometric parameters using the method of Scorza \& Bender (1995) as implemented by Morelli et al. (2004). This method is based on the assumption that the isophotal diskiness is the result of the superimposition of a spheroidal component (which is either a host elliptical galaxy or a bulge component) and an inclined exponential disk. The two components are assumed to have both perfectly elliptical isophotes with constant but different ellipticities.

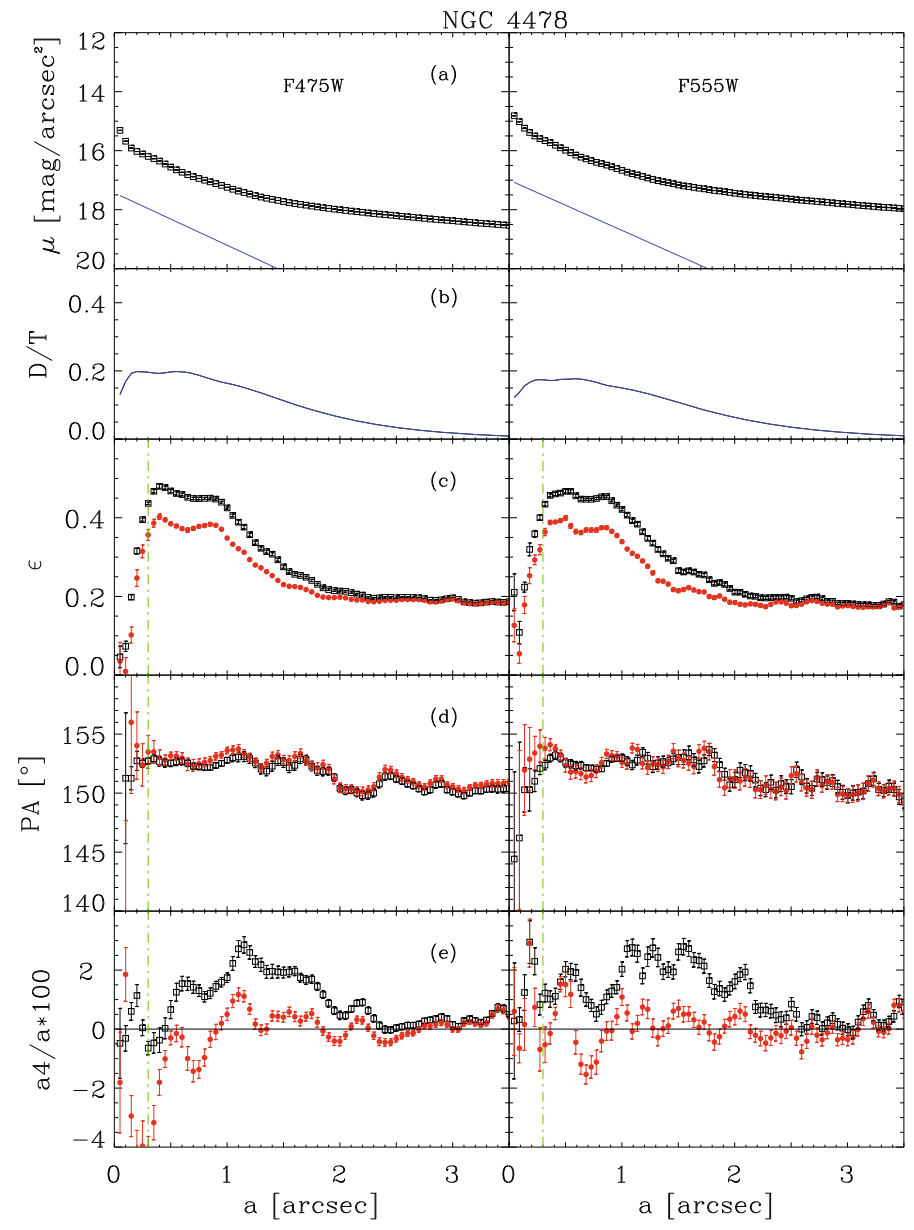

Fig. 3. Same as in Fig. 2, but for NGC 4478. The results of the photometric decomposition of the WFPC2/F814W image are in Morelli et al. (2004).

To properly derive the photometric parameters of the nuclear disks, it is important to account for the HST point-spread function (PSF). The adopted PSF model was calculated with the TINYTIM package by taking into account the instrumental setup and position of the nuclear disk on the given image (Krist \& Hook 1999). To restore the images from the effects of the PSF, they were deconvolved with the Richardson-Lucy method (Richardson 1972; Lucy 1974) by applying the IRAF task LUCY for a number of iterations between 3 and 6. A larger number of iterations was not found to improve the restoration of the geometrical parameters of the isophotes as discussed in detail by Michard (1996) with extensive experiments on the ground and HST-based images. The image noise was, instead, amplified and errors in the ellipticity and $a_{4}$ Fourier coefficients are enlarged. This effect is illustrated in Fig. 5 for the WFPC2/F555W image of NGC 4458. The average surface brightness in the galaxy nucleus and the image noise are plotted as a function of the number of iterations performed with the deconvolution algorithm. The average surface brightness of the galaxy was measured in circular apertures of radii 2,3 , and 6 pixels, respectively and centered on the nucleus. They correspond approximately to 3,5 , and 10 times the standard deviation of the best-fit Gaussian model of the PSF. The noise was estimated by measuring the standard deviation in the surface brightness level for a number of empty regions at the edges of the field of view. After 6 iterations, a negligible rise $(<2 \%)$ in the surface brightness is observed even in the innermost aperture, which is the most sensitive to 


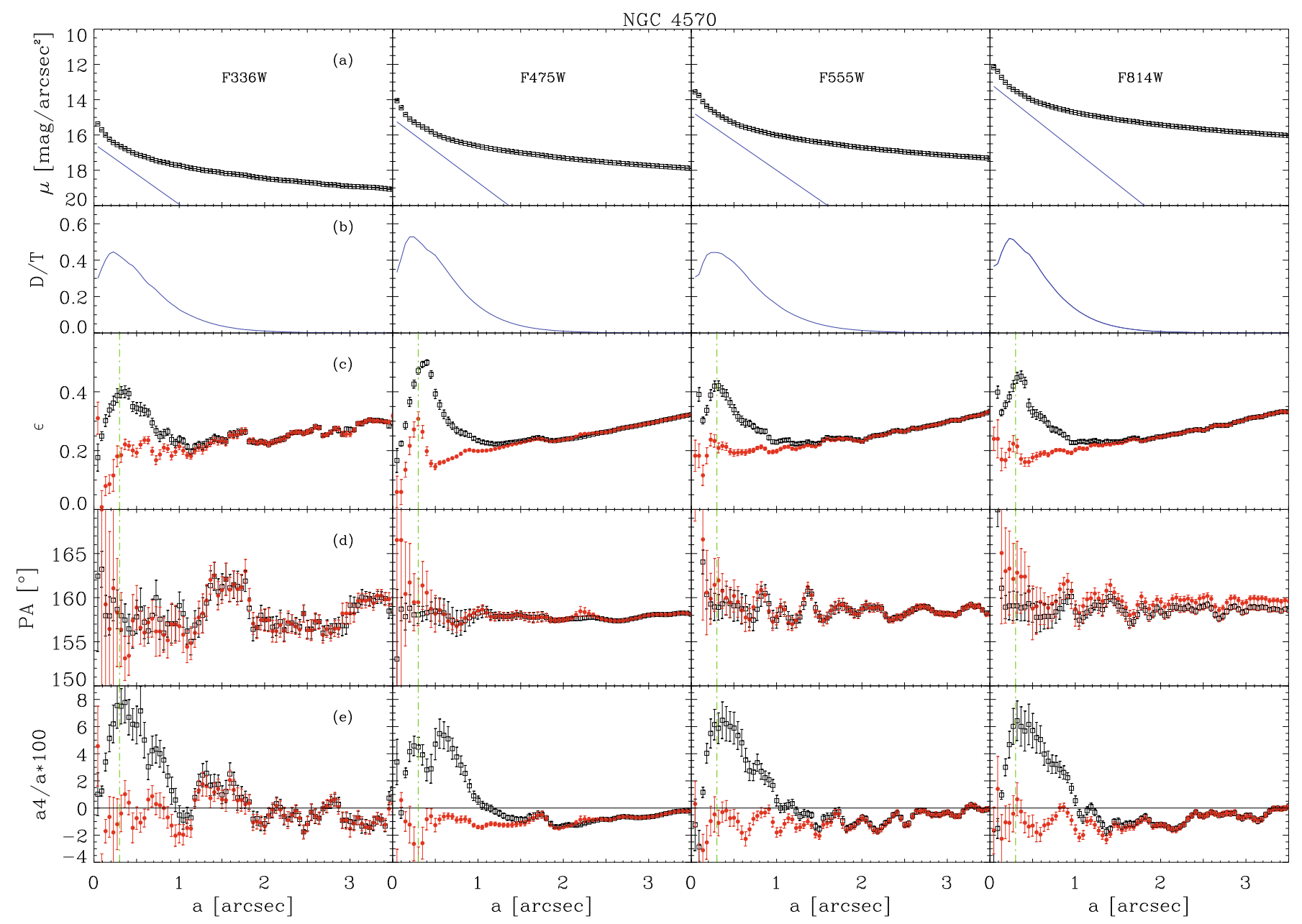

Fig. 4. Same as in Fig. 2, but for the WFPC2/F336W, ACS/F475W, WFPC2/F555W, and WFPC2/F814W images of NGC 4570.

the deconvolution effect. In contrast, the noise level increases continuously at each iteration. This increases the uncertainties in the $a_{4}$ Fourier coefficients, which are critical for recovering the structural parameters of the NSD with the method of photometric decomposition by Scorza \& Bender (1995). The radial profiles of the $a_{4}$ Fourier coefficients measured in the original WFPC2/F555W image of NGC 4458 as well as in the images resulting after 6 and 20 deconvolution iterations are shown in Fig. 5. The redistribution of the galaxy light caused by the deconvolution is characterized by an enhancement of the galaxy diskiness in the inner $1^{\prime \prime}$. The $a_{4}$ Fourier coefficients measured after 6 and 20 iterations have the same radial trend and are consistent within the errors. However, the $a_{4}$ errorbars increased by a factor of 3 after 20 iterations. We found that after 40 Richardson-Lucy iterations the scatter and errorbars of the $a_{4}$ Fourier coefficients are sufficiently large to blur the peak in the $a_{4}$ radial profile and significantly reduce the radial range along which the diskiness is observed. This prevents us obtaining accurate and reliable values of the NSD parameters.

The rms tracking error is negligible with respect to the PSF FWHM (0.'07), thus it does not affect the image quality and the measurement of the structural parameters of the NSDs. To this aim, no correction for telescope jitter was necessary.

The Scorza \& Bender (1995) method consists of the iterative subtraction of a thin disk model characterized by an exponential surface-brightness profile of central surface brightness $\mu_{0}$, scalelength $h$, axial ratio $b / a$, and position angle PA. The disk inclination was calculated to be $i=\arccos (b / a)$. The position angle of each nuclear disk was found to be constant within $1^{\circ}$ for all the images of the same galaxy (Table 2). Adopting this value, the remaining disk parameters are adjusted until the departures from perfect ellipses are minimized (i.e., $a_{4} \approx 0$ ).

Ferrarese et al. (2006) measured in HST/ACS images the isophotal parameters of sample of early-type members of the Virgo cluster (including NGC 4458, NGC 4478, and NGC 4570). Inside $r<0.1 r_{\mathrm{e}}$, there is a trend for the mean $a_{4}$ to increase as galaxies become fainter. On large scales $\left(0.1 r_{\mathrm{e}}<r<\right.$ $\left.r_{\mathrm{e}}\right)$, the isophotes are consistent with having a constant shape. In this radial range, most (but not all) of the observed galaxies exhibit almost elliptical isophotes. These results agree with the previous findings based on HST photometry (van den Bosch et al. 1994; Lauer et al. 1995; Verdoes Kleijn et al. 1999; Rest et al. 2001; but see also Kormendy et al. 2009). We therefore decided to adjust the NSD parameters until the isophotes of the host spheroid had the same shape observed outside the inner region where $a_{4}>0$. We adopted $a_{4} \simeq 0.01$ for NGC 4458, $a_{4} \simeq 0$ for NGC 4478, and $a_{4} \simeq-0.01$ for NGC 4570, respectively.

For each disk model, the disk-free image of the galaxy was obtained by subtracting the disk model from the galaxy image. The isophotal analysis of the disk-free image was performed using the IRAF task ELLIPSE. The photometric decomposition was performed independently for each image. The best-fit values of $\mu_{0}, h$, and $b / a$ and their $68 \%$ confidence-level errors were derived in a similar way to those in Morelli et al. (2004) and are listed in Table 2. The comparison between the isophotal parameters of the galaxies measured before and after the subtraction of 
L. Morelli et al.: Multiband photometric decomposition of nuclear stellar disks

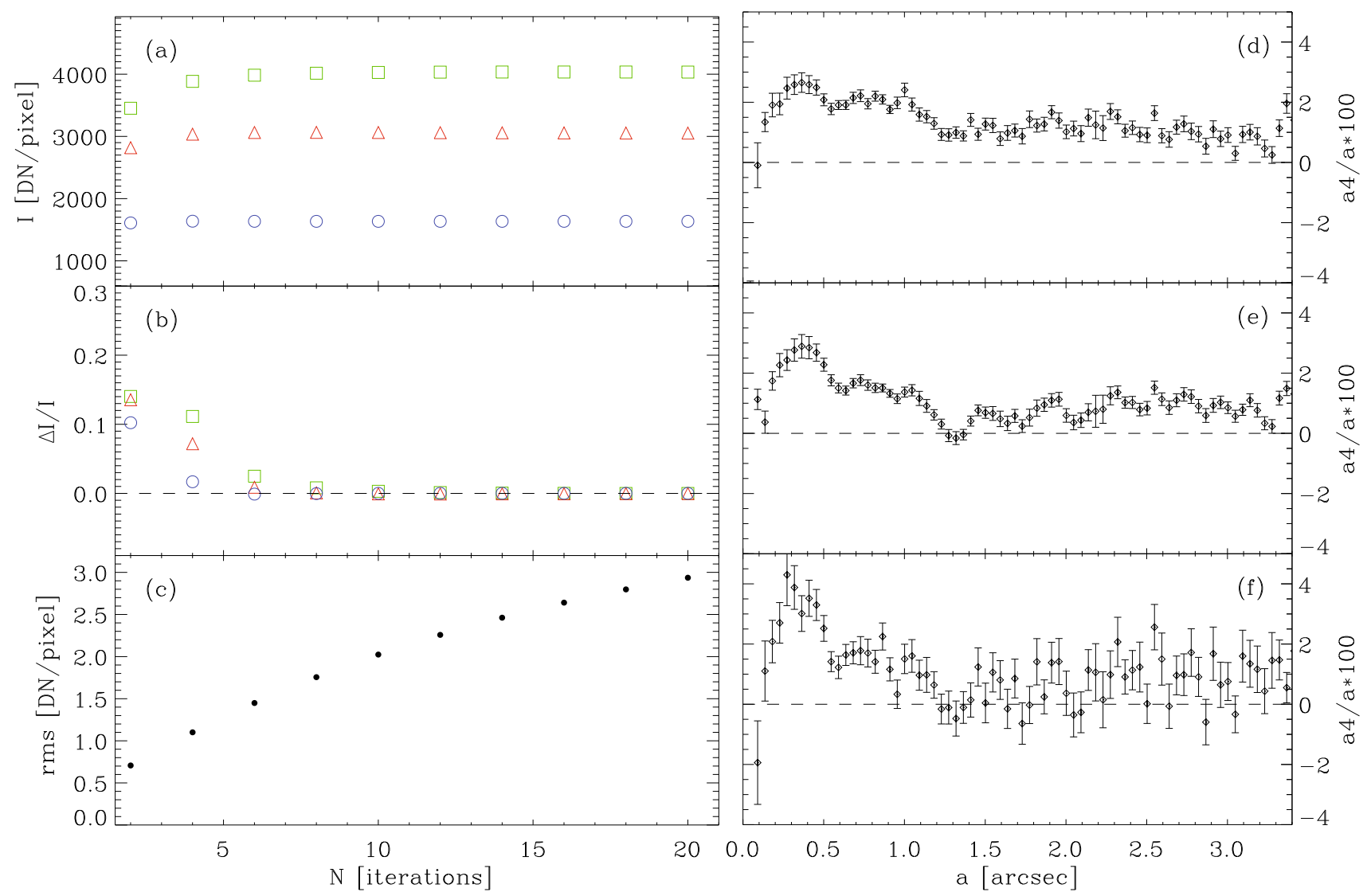

Fig. 5. Deconvolution of the WFPC2/F555W image of NGC 4458 with the Richardson-Lucy algorithm. The left panels show a) the average surface brightness per pixel in the galaxy nucleus, b) its relative increase, and c) the noise level of the image plotted as a function of the number of the iterations of the deconvolution process. The different symbols refer to the values measured in circular apertures with radii of 2 (green squares), 3 (red triangles), and 6 (blue circles) pixels centered on the galaxy nucleus, respectively. The relative increase in surface brightness at iteration 1 is calculated with respect to the original undeconvolved image. The noise level (filled circles) of the image was measured after each iteration in the same empty regions at the edges of the field of view. The right panels show the radial profiles of the fourth cosine Fourier coefficients measured in the d) original image, and in the deconvolved image after e) 6 and f) 20 iterations.

Table 2. Photometric parameters of the nuclear stellar disks.

\begin{tabular}{|c|c|c|c|c|c|c|c|c|}
\hline \multirow{3}{*}{$\begin{array}{c}\text { Galaxy } \\
\text { (1) }\end{array}$} & \multirow{3}{*}{$\begin{array}{l}\text { Filter } \\
\text { (2) }\end{array}$} & \multirow{3}{*}{$\begin{array}{c}\mu_{0} \\
{\left[\mathrm{mag} \operatorname{arcsec}^{-2}\right]} \\
\text { (3) }\end{array}$} & \multicolumn{2}{|l|}{$h$} & \multirow{3}{*}{$\begin{array}{c}i \\
{\left[{ }^{\circ}\right]} \\
(5)\end{array}$} & \multirow{3}{*}{$\begin{array}{l}\text { PA } \\
{\left[{ }^{\circ}\right]} \\
(6)\end{array}$} & \multirow{3}{*}{$\begin{array}{c}L \\
{\left[10^{6} L_{\odot}\right]} \\
(7)\end{array}$} & \multirow{3}{*}{$\begin{array}{l}\text { Ref. } \\
\text { (8) }\end{array}$} \\
\hline & & & {$\left[{ }^{\prime \prime}\right]$} & {$[\mathrm{pc}]$} & & & & \\
\hline & & & \multicolumn{2}{|c|}{ (4) } & & & & \\
\hline \multirow[t]{3}{*}{ NGC 4458} & $F 475 W$ & $16.0_{-1.0}^{+0.3}$ & $0.24_{-0.07}^{+0.30}$ & $15_{-4}^{+18}$ & $84_{-13}^{+6}$ & $7 \pm 1$ & $2.7_{-0.5}^{+3.3}$ & 1 \\
\hline & $F 555 W$ & $15.8_{-0.9}^{+0.2}$ & $0.24_{-0.04}^{+0.02}$ & $15_{-2}^{+1}$ & $82_{-6}^{+8}$ & $7 \pm 1$ & $3.3_{-0.5}^{+3.5}$ & 1 \\
\hline & $F 814 W$ & $14.3_{-0.7}^{+0.7}$ & $0.18_{-0.04}^{+0.99}$ & $11_{-2}^{+6}$ & $83_{-9}^{+7}$ & $7 \pm 1$ & $3.0_{-2.7}^{+3.0}$ & 2 \\
\hline \multirow[t]{3}{*}{ NGC 4478} & $F 475 W$ & $17.5_{-0.3}^{+1.4}$ & $0.55_{-0.34}^{+0.54}$ & $34_{-21}^{+32}$ & $78_{-12}^{+12}$ & $153 \pm 1$ & $6.8_{-2.1}^{+0.9}$ & 1 \\
\hline & $F 555 W$ & $17.1_{-0.5}^{+1.3}$ & $0.63_{-0.25}^{+0.95}$ & $38_{-15}^{+58}$ & $78_{-11}^{+12}$ & $153 \pm 1$ & $10.5_{-3.6}^{+6.2}$ & 1 \\
\hline & $F 814 W$ & $15.8_{-0.8}^{+1.5}$ & $0.66_{-0.30}^{+0.54}$ & $40_{-19}^{+33}$ & $80_{-10}^{+10}$ & $153 \pm 1$ & $17.0_{-6.3}^{+5.6}$ & 2 \\
\hline \multirow[t]{5}{*}{ NGC 4570} & $F 336 \mathrm{~W}$ & $16.6_{-1.0}^{+0.7}$ & $0.31_{-0.04}^{+0.32}$ & $19_{-2}^{+20}$ & $83_{-6}^{+7}$ & $158 \pm 1$ & $4.5_{-2.5}^{+7.9}$ & 1 \\
\hline & $F 475 W$ & $15.3_{-0.9}^{+0.3}$ & $0.27_{-0.07}^{+0.32}$ & $16_{-4}^{+20}$ & $78_{-7}^{+12}$ & $158 \pm 1$ & $13.7_{-1.4}^{+6.9}$ & 1 \\
\hline & $F 555 W$ & $14.8_{-1.0}^{+0.7}$ & $0.32_{-0.05}^{+0.40}$ & $19_{-3}^{+25}$ & $83_{-3}^{+7}$ & $158 \pm 1$ & $12.9_{-5.0}^{+16.0}$ & 1 \\
\hline & $F 555 W$ & 14.8 & $\ldots$ & 23 & $\ldots$ & $\ldots$ & 19 & 3 \\
\hline & $F 814 W$ & $13.2_{-0.9}^{+0.9}$ & $0.28_{-0.08}^{+0.35}$ & $17_{-5}^{+22}$ & $81_{-6}^{+9}$ & $158 \pm 1$ & $22.4_{-10.0}^{+22.0}$ & 1 \\
\hline
\end{tabular}

Notes. Column (1): Galaxy name. Column (2): filter name. Column (3): disk central surface-brightness in the Vega magnitude system. Column (4): disk scale length. A distance of $12.6 \mathrm{Mpc}$ was adopted for all the sample galaxies. Column (5): disk inclination. Column (6): disk position angle. Column (7): disk luminosity. Column (8): reference paper for the photometric decomposition.

References. 1 = This work, 2 = Morelli et al. (2004), 3 = Scorza \& van den Bosch (1998). 
the best-fit model of their nuclear disk are shown in Figs. 2-4. The surface-brightness radial profiles of the nuclear disks and the radial profile of the light fraction $D / T$ that they contribute to the galaxy luminosity are also plotted.

The $a_{4}$ Fourier coefficients observed in the three galaxies drop to zero at small radii $(<0,3)$, where the galaxy isophotes become rounder $(\epsilon<0.4)$ and perfectly elliptical (Figs. 2-4). The NSD models have elliptical isophotes of higher ellipticity $(\epsilon \simeq$ 0.8 ; Table 2). When they are subtracted from the galaxy images, the innermost residual isophotes becomes boxy $\left(a_{4}<0\right)$. This is an artefact of the Richardson-Lucy deconvolution method. As discussed by Michard (1996), the algorithm fails to recover the geometrical properties of the galaxy cores. In particular, the restored ellipticity is underestimated and decreases to zero towards the center (see also Lucy 1994). van den Bosch et al. (1998) place a lower limit of 0 !' 3 for recovering reliable geometric parameters in HST/WFPC2 images, and Scorza \& van den Bosch (1998) adopted this limit in their nuclear disk decomposition of NGC 4342 and NGC 4570. We assume that the results obtained by van den Bosch et al. (1998) are directly applicable to our case: because we study images obtained with similar or longer integration times, galaxies with shallower surface-brightness profiles and NSDs with equal or larger scale lengths. The decomposition results do not depend on the surface-brightness distribution in this region. Figure 6 shows the shape of the isophotes of the sample galaxies before and after the subtraction of the best-fit model of the NSD. The WFPC2/F555W images are considered here as an example. The diskiness of the galaxy isophotes disappears after the model subtraction. The same behaviour is also observed in the other available images.

$N G C$ 4458. To find the best-fit model of NGC 4458, we adjusted the disk parameters until the galaxy isophotes in the inner $1^{\prime \prime}$ have $a_{4} \simeq 0.01$ instead of being perfectly elliptical (Fig. 2). The scale length, inclination, and position angle of the nuclear disk measured in the ACS/F475W and WFPC2/F555W passbands are consistent within the errors. They are also consistent within the errors with the values measured by Morelli et al. (2004) in the WFPC2/F814W image (Fig. 7). The mean values of the parameters are $\langle h\rangle=13 \mathrm{pc},\langle i\rangle=83^{\circ}$, and $\langle\mathrm{PA}\rangle=7^{\circ}$, respectively. It is the smallest and least luminous NSD $\left(L_{\mathrm{F} 814 \mathrm{~W}}=3 \times 10^{6} L_{\odot}\right)$ of the sample. Its light contribution peaks at 0.3 , where $D / T$ ranges from about 0.4 (ACS/F475W) to 0.2 (WFPC2/F555W) depending on the passband (Fig. 2).

$N G C$ 4478. The scale length, inclination, and position angle of the nuclear disk in the ACS/F475W and WFPC2/F555W passbands are consistent within the errors with the WFPC2/F814W values by Morelli et al. (2004, Fig. 7). The mean values of the parameters are $\langle h\rangle=37 \mathrm{pc},\langle i\rangle=78^{\circ}$, and $\langle\mathrm{PA}\rangle=153^{\circ}$, respectively. The nuclear disk contributes a maximum $D / T=0.2$ in the radial range between $0 \prime^{\prime} 2$ and $0{ }^{\prime} 6$ from the center (Fig. 3). It is one of the largest and more luminous NSDs $\left(L_{\mathrm{F} 814 \mathrm{~W}}=\right.$ $\left.2 \times 10^{7} L_{\odot}\right)$ known to date.

$N G C 4570$. To find the best-fit model of NGC 4570, we adjusted the disk parameters until the galaxy isophotes in the inner $1^{\prime \prime}$ have $a_{4} \simeq-0.01$ instead of being perfectly elliptical (Fig. 4). In this way, we account for the isophotal shape of the galaxy bulge, which is characterized by an increasing boxiness to $a_{4} \simeq$ -0.01 going from $3^{\prime \prime}$ to $1^{\prime \prime}$. The central surface-brightness, scale length, and inclination of the NSD were already obtained from the analysis of the WFPC2/F555W image by van den Bosch (1998). We also decided to perform the photometric decomposition using our method too. Homogeneous decompositions and errors in the fitted parameters are required to properly compare

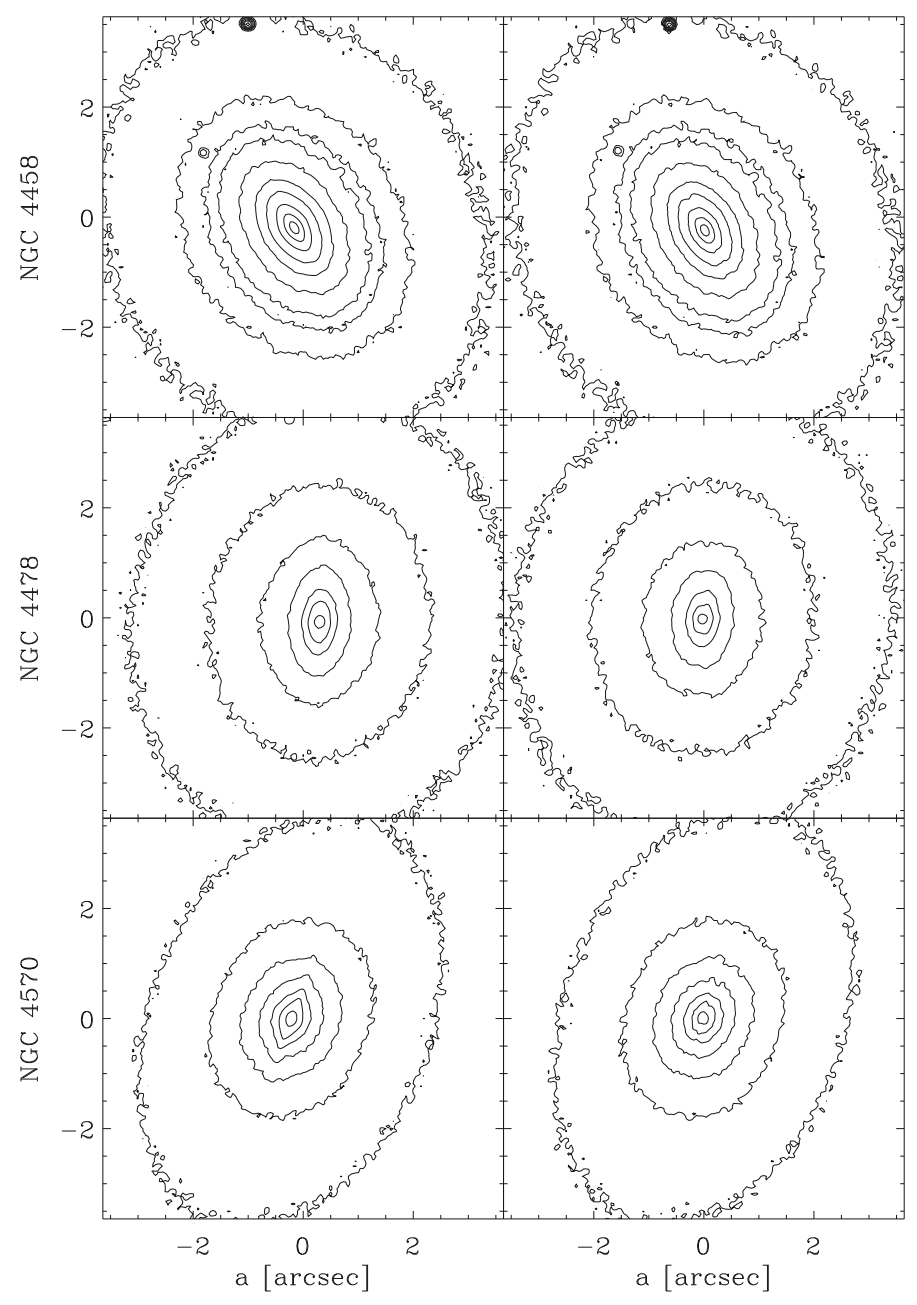

Fig. 6. Contour plots of the WFPC2/F555W deconvolved images of NGC 4458, NGC 4478, and NGC 4570 before (left panels) and after (right panels) the nuclear disk subtraction. Scales are in arcseconds, and orientations are the same as in Fig. 1.

the structural parameters of the NSD in the different passbands. Our results agree within the errors with those by van den Bosch (1998). Moreover, the averaged scale length, inclination, and position angle remain constant within the errors in all the observed passbands (Fig. 7). Their mean values are $\langle h\rangle=18 \mathrm{pc},\langle i\rangle=81^{\circ}$, and $\langle\mathrm{PA}\rangle=158^{\circ}$, respectively. The disk luminosity ranges from $5 \times 10^{6}(\mathrm{WFPC} 2 / \mathrm{F} 336 \mathrm{~W})$ to $2 \times 10^{7} L_{\odot}(\mathrm{WFPC} 2 / \mathrm{F} 814 \mathrm{~W})$. Its maximum $D / T \simeq 0.5$ is observed at about 0.'3 (Fig. 4 ).

The trend of the central surface brightness in the different passbands is similar for all the measured NSDs. The value of the central surface brightness is constant within the errors in the ACS/F475W and WFPC2/F555W passbands and decreases by 1.6 mag $\operatorname{arcsec}^{-2}$ in the WFPC2/F814W band. The WFPC2/F336W image was available only for NGC 4570. The central value of the surface brightness in this filter is about $1.3 \mathrm{mag} \operatorname{arcsec}^{-2}$ greater than that in $\mathrm{ACS} / \mathrm{F} 475 \mathrm{~W}$ passband. In Table 3, we report the colors NSDs and of the their host spheroids. The colors of the spheroid were measured along the disk major axis at a distance of 10 scale lengths on both sides of the center, where the contribution of the NSD to the total light is negligible (Figs. 2-4). The conversion to the Johnson system was calculated using SYNPHOT in IRAF. Since this correction depends on the spectral energy distribution of the object, it was calculated using the Kinney et al. (1996) spectral templates. The 
L. Morelli et al.: Multiband photometric decomposition of nuclear stellar disks

Table 3. Colors of the NSDs and their host spheroids.

\begin{tabular}{ccccccc}
\hline \hline \multirow{2}{*}{ Color } & \multicolumn{2}{c}{ NGC 4458 } & \multicolumn{2}{c}{ NGC 4478 } & \multicolumn{2}{c}{ NGC 4570 } \\
& NSD & Spheroid & NSD & Spheroid & NSD & Spheroid \\
\hline$U-B$ & $\ldots$ & $\ldots$ & $\ldots$ & $\ldots$ & 1.1 & 0.8 \\
$B-V$ & 0.6 & 1.0 & 0.8 & 1.1 & 0.9 & 1.0 \\
$V-I$ & 1.4 & 1.1 & 1.2 & 1.1 & 1.5 & 1.2 \\
\hline
\end{tabular}

Notes. The conversion to the Johnson system has been calculated as $U-B=[F 336 W-F 475 W]-0.21 ; B-V=[F 475 W-F 555 W]+0.36$; $V-I=[F 555 W-F 814 W]-0.05$.

resulting colors are similar to those measured in galaxies hosting a NSD (NGC 4128, NGC 4621, and NGC 5308, Krajnović \& Jaffe 2004; NGC 4486A, Kormendy et al. 2005.) The large uncertainties in the central surface brightnesses are intrinsic to the photometric decomposition method. This prevented us from performing color analysis as a consistency check with the measurement of age and metallicity performed by van den Bosch et al. (1998), Morelli et al. (2004), and Krajnović \& Jaffe (2004) and based on the line-strength indices.

The scale length, inclination, and position angle derived for each NSD in the different filters are constant within the errors, independently of their values and the properties of their host galaxy. The nuclear disk has the same position angle as the inner part of the host spheroid.

\section{Discussion and conclusions}

We have investigated the photometric properties of the NSDs of three early-type galaxies in the Virgo cluster, NGC 4458, NGC 4478, and NGC 4570 by analyzing the WFPC2 and ACS images of their nuclei available in the HST Science Archive. The images were unsharp masked and their visual inspection suggested that the size, orientation, and location of each NSD was independent of the observed passband. To verify this, we derived the central surface brightness, scale length, inclination, and position angle of each NSD in all the passbands by applying the photometric decomposition method of Scorza \& Bender (1995). Some of the images had already been analyzed by van den Bosch et al. (1998, WFPC2/F555W for NGC 4570) and Morelli et al. (2004, WFPC2/F814W for NGC 4458 and NGC 4478). Since we adopted an identical procedure to that of Morelli et al. (2004), we relied on their WFPC2/F814W decomposition of NGC 4458 and NGC 4478 and repeated the WFPC2/F555W analysis of NGC 4570 to build homogeneous set of photometric parameters and corresponding errors for all the NSDs in all the passbands.

The structural parameters (i.e., scale length, inclination, and position angle) of each NSD are constant within the errors in all the observed passbands, independently of their values and the properties of their host spheroid. This implies an absence of color gradients in nuclear disks and can be used to constrain their star formation processes. NSDs are considered to be the end result of the formation of stars from gas of external (i.e., accreted from galaxy neighborhoods or captured during a merger with a gas rich-companion; e.g., NGC 4458, NGC 4478 Morelli et al. 2004) or internal origin (i.e., conveyed from outer galactic regions by a bar; e.g., NGC 4570 van den Bosch \& Emsellem 1998) piled up in the galaxy nucleus. Thus independent of the way in which the gas was originally accumulated towards the center, the dissipational formation process has to be invoked to account for the dynamically cold structure of the nuclear disks.

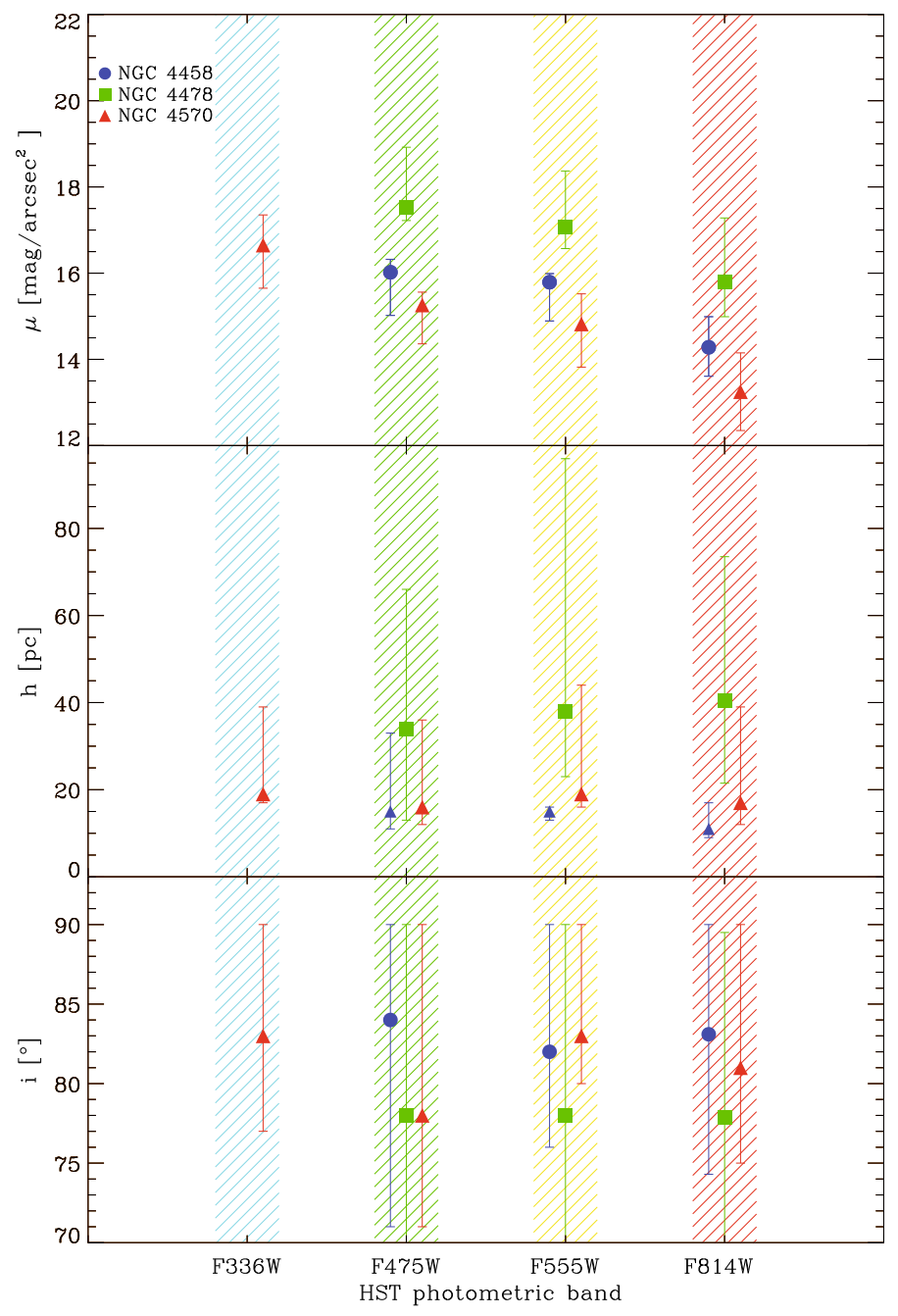

Fig. 7. Photometric parameters of the nuclear stellar disks of NGC 4458 (blue circles), NGC 4478 (green squares), and NGC 4570 (red triangles) in the WFPC2/F336W, ACS/F475W, WFPC2/F555W, and WFPC2/F814W passbands.

This is hard to explain if NSDs assembled from already formed stars. We interpret the absence of color gradients in NDSs as the signature of a star formation event that occurred homogeneously over the entire disk. Although our sample is admittedly very small, an inside-out formation scenario (Muñoz-Mateos et al. 2007) seems to be ruled out in NSDs given that this would produce radial population gradients that are not observed.

Acknowledgements. We would like to thank Enzo Brocato, Valentin Ivanov, Jairo Méndez-Abreu and Lodovico Coccato for their useful suggestions. L.M. is supported by grant CPDR061795/06 from Padua University. M.C. gratefully acknowledges the European Southern Observatory (ESO) for support via ESO Studentship program at the ESO Research Facilities in Santiago. This research has been made possible by support from Padua University through grants CPDA068415/06, CPDA089220/08, and CPDR095001/09 and from Istituto Nazionale di Astrofisica (INAF) through grant PRIN2005/32. This research has made use of the Lyon Extragalactic Database (LEDA) and NASA/IPAC Extragalactic Database (NED).

\section{References}

Athanassoula, E., Lambert, J. C., \& Dehnen, W. 2005, MNRAS, 363, 496 Baggett, S., McMaster, M., Biretta, J., et al. 2002, WFPC2 Data Handbook, Version 4.0, STScI, Baltimore

Balcells, M., Graham, A. W., \& Peletier, R. F. 2007, ApJ, 665, 1084

Barnes, J. E., \& Hernquist, L. 1996, ApJ, 471, 115 
Bertola, F., \& Corsini, E. M. 1999, in Galaxy Interactions at Low and High Redshift, ed. J. E. Barnes, \& D. B. Sanders (San Francisco: ASP), IAU Symp., 186, 149

Bertola, F., Corsini, E. M., Vega Beltrán, J. C., et al. 1999, ApJ, 519, L127

Carollo, C. M., Danziger, I. J., Rich, R. M., \& Chen, X. 1997, ApJ, 491, 545

de Vaucouleurs, G., de Vaucouleurs, A., Corwin, H. G., et al. 1991, Third Reference Catalogue of Bright Galaxies (Berlin: Springer) (RC3)

Dotti, M., Colpi, M., Haardt, F., \& Mayer, L. 2007, MNRAS, 379, 956

Eliche-Moral, M. C., Gonzalez-Garcia, A. C., Balcells, M., et al. 2009, in Hunting for the Dark: The Hidden Side of Galaxy Formation, ed. V. P. Debattista, \& C. C. Popescu, AIP Conf. Ser., 1240, 237

Emsellem, E., Monnet, G., Bacon, R., \& Nieto, J.-L. 1994, A\&A, 285, 739

Emsellem, E., Bacon, R., Monnet, G., \& Poulain, P. 1996, A\&A, 312, 777

Emsellem, E., Cappellari, M., Krajnović, D., et al. 2007, MNRAS, 379, 401

Falcón-Barroso, J., Bacon, R., Bureau, M., et al. 2006, MNRAS, 369, 529

Ferrarese, L., Côté, P., Jordán, A., et al. 2006, ApJS, 164, 334

Halliday, C., Davies, R. L., Kuntschner, H., et al. 2001, MNRAS, 326, 473

Holtzman, J. A., Burrows, C. J., Casertano, S., et al. 1995, PASP, 107, 1065

Hopkins, P. F., \& Quataert, E. 2010, MNRAS, 405, L41

Jedrzejewski, R. I. 1987, MNRAS, 226, 747

Kinney, A. L., Calzetti, D., Bohlin, R. C., et al. 1996, ApJ, 467, 38

Kormendy, J., \& Bender, R. 1996, ApJ, 464, L119

Kormendy, J., Dressler, A., Byun, Y. I., et al. 1994, in Dwarf Galaxies, ed. G. Meylan, \& P. Prugniel (Garching: ESO), ESO Conf. Ser., 49, 147

Kormendy, J., Bender, R., Richstone, D., et al. 1996, ApJ, 459, L57

Kormendy, J., Gebhardt, K., Fisher, D. B., et al. 2005, AJ, 129, 2636

Kormendy, J., Fisher, D. B., Cornell, M. E., \& Bender, R. 2009, ApJS, 182, 216

Krajnović, D., \& Jaffe, W. 2004, A\&A, 428, 877

Krist, J., \& Hook, R. 1999, STIS Instrument Handbook Version 4.0, STScI, Baltimore

Kuntschner, H., Emsellem, E., Bacon, R., et al. 2006, MNRAS, 369, 497

Lauer, T. R., Ajhar, E. A., Byun, Y.-I., et al. 1995, AJ, 110, 2622

Ledo, H. R., Sarzi, M., Dotti, M., Khochfar, S., \& Morelli, L. 2010, MNRAS, accepted [arXiv: 1005.2524]

Lucy, L. B. 1974, AJ, 79, 745

Lucy, L. B. 1994, in The Restoration of HST Images and Spectra - II, ed. R. J. Hanisch, \& R. L. White, STScI, Baltimore, 79

Maraston, C. 1998, MNRAS, 300, 872

McDermid, R. M., Emsellem, E., Shapiro, K. L., et al. 2006, MNRAS, 373, 906
McMaster, M., Biretta, J., et al. 2008, WFPC2 Instrument Handbook, Version 10.0, STScI, Baltimore

Michard, R. 1996, A\&AS, 117, 583

Morelli, L., Halliday, C., Corsini, E. M., et al. 2004, MNRAS, 354, 753

Morelli, L., Pompei, E., Pizzella, A., et al. 2008, MNRAS, 389, 341

Muñoz-Mateos, J. C., Gil de Paz, A., Boissier, S., et al. 2007, ApJ, 658, 1006

Pavlovsky, C., Biretta, J., Bohlin, R., et al. 2004, ACS Instrument Handbook, Version 5.0, STScI, Baltimore

Pavlovsky, C., Koekemoer, A., Mack, J., et al. 2006, ACS Data Handbook, Version 5.0, STScI, Baltimore

Peletier, R. F., Falcón-Barroso, J., Bacon, R., et al. 2007, MNRAS, 379, 445

Pizzella, A., Corsini, E. M., Morelli, L., et al. 2002, ApJ, 573, 131

Ravindranath, S., Ho, L. C., Peng, C. Y., Filippenko, A. V., \& Sargent, W. L. W. 2001, AJ, 122, 653

Rest, A., van den Bosch, F. C., Jaffe, W., et al. 2001, AJ, 121, 2431

Richardson, W., H. 1972, Opt. Soc. Am. J., 62, 476

Rix, H., \& White, S. D. M. 1990, ApJ, 362, 52

Sandage, A., \& Tammann, G. A. 1981, A Revised Shapley-Ames Catalog of Bright Galaxies (Washington D.C.: Carnegie Institution)

Scorza, C., \& Bender, R. 1995, A\&A, 293, 20

Scorza, C., \& van den Bosch, F. C. 1998, MNRAS, 300, 469

Seifert, W., \& Scorza, C. 1996, A\&A, 310, 75

Seth, A. C., Dalcanton, J. J., Hodge, P. W., \& Debattista, V. P. 2006, AJ, 132, 2539

Simões Lopes, R. D., Storchi-Bergmann, T., de Fátima Saraiva, M., \& Martini, P. 2007, ApJ, 655, 718

Sirianni, M., Jee, M. J., Benítez, N., et al. 2005, PASP, 117, 1049

Thomas, D., Maraston, C., \& Bender, R. 2003, MNRAS, 339, 897

Tran, H. D., Tsvetanov, Z., Ford, H. C., et al. 2001, AJ, 121, 2928

Tully, R. B. 1988, Science, 242, 310

van den Bosch, F. C. 1998, ApJ, 507, 601

van den Bosch, F. C., \& Emsellem, E. 1998, MNRAS, 298, 267

van den Bosch, F. C., Ferrarese, L., Jaffe, W., Ford, H. C., \& O’Connell, R. W. 1994, AJ, 108, 1579

van den Bosch, F. C., Jaffe, W., \& van der Marel, R. P. 1998, MNRAS, 293, 343 van Dokkum, P. G. 2001, PASP, 113, 1420

Verdoes Kleijn, G. A., Baum, S. A., de Zeeuw, P. T., \& O’Dea, C. P. 1999, AJ, 118, 2592

Worthey, G. 1994, ApJS, 95, 107 